\title{
Les essais de phase I, après mais aussi avant
}

\author{
Phase I Trials, Afterwards but Also Before
}

\section{J.-D. Zeitoun · R. Lasseur}

(C) Lavoisier SAS 2016

L'actualité récente a brutalement porté à la connaissance du grand public mondial l'existence et la réalité des essais de phase I. En janvier 2015, un essai de ce type mené à Rennes a pris un tour tragique avec l'hospitalisation en fin d'essai de six personnes pour troubles neurologiques sévères, dont l'une est rapidement décédée. Les cinq autres personnes ont pu quitter l'hôpital et on ne sait actuellement pas si elles conserveront des séquelles définitives de leur hospitalisation inattendue. L'écho de cet accident sériel a été mondial, relayé par les plus grandes revues scientifiques [1] et les blogs internationaux les plus influents [2]. Les volontaires sains concernés participaient à un essai sur une molécule d'un laboratoire portugais qui visait une indication future dans les troubles anxieux et la douleur. Diverses enquêtes - administrative, réglementaire et judiciaire - sont en cours, dont les résultats ne sont pas connus à l'écriture de cet éditorial, à l'exception d'un rapport préliminaire de l'Inspection Générale des Affaires Sociales (IGAS) qui sera abordé plus bas.

Le système de phases des essais thérapeutiques constitue le squelette du développement clinique des médicaments. Cette ossature quasi invariante n'est pas un produit académique ni une invention industrielle mais elle prend son origine dans le désastre historique de la thalidomide. À la fin des années 1950, un laboratoire allemand commercialisa ce médicament pour traiter les sensations négatives liées à la grossesse. Le produit connut un grand succès commercial

\footnotetext{
J.-D. Zeitoun $(\bowtie)$

Proctologie médico-interventionnelle, groupe hospitalier Diaconesses Croix Saint-Simon, site Avron, 125 rue d'Avron, F75020 Paris

e-mail : jdzeitoun@yahoo.fr
}

Gastroentérologie et nutrition, hôpital Saint-Antoine, 184 rue du Faubourg Saint-Antoine, F-75012 Paris

Inato, 113 rue Saint-Maur, F-75011 Paris

\section{R. Lasseur $(\square)$}

Toxicologue associé, Animal \& Co, 30 rue Pré Gaudry, F-69007

Lyon

e-mail : r.lasseur@animalco.fr mais ne fut pas approuvé aux États-Unis, grâce à la préscience d'une pharmacologue canadienne qui venait de prendre son poste à la Food and Drug Administration, Frances Kelsey, décédée récemment à l'âge de 101 ans. Frances Kelsey estimait en effet que la sécurité de la molécule n'était pas suffisamment établie et le médicament ne fut jamais autorisé sur le marché américain. Entre temps, il provoqua le décès de plus de 2000 bébés et des malformations chez au moins 10000 autres avant d'être retiré des pharmacies entre 1961 et 1962. Bien que leurs concitoyens aient été épargnés, les parlementaires américains ont mis en œuvre dès 1962 des réformes profondes au sein de la Food and Drug Administration. La nouvelle législation visait essentiellement à conférer à l'agence l'autorité d'exiger des preuves d'efficacité des molécules qui lui étaient soumises pour approbation (les preuves de sécurité étaient exigibles depuis 1937). C'est de là qu'a été construite la structure en trois phases des essais cliniques qui succèdent à l'expérimentation animale [3].

Les essais de phase I font appel à un faible nombre d'individus. Sauf exception, comme le cancer, il s'agit de volontaires non atteints par la pathologie que vise la molécule d'intérêt. Ils sont en général entre quelques dizaines et une centaine, souvent des hommes pour éviter le risque de grossesse. L'objectif principal des essais de phase I est d'éliminer une toxicité majeure et de caractériser les toxicités mineures des médicaments testés.

Que nous disent les différentes informations communiquées sur les causes du drame rennais ? Très peu de choses en réalité. La note d'étape de l'IGAS souligne trois manquements qui peuvent être résumés comme suit : défaut d'information sur l'état du volontaire hospitalisé et poursuite de l'administration du produit à d'autres volontaires, défaut d'information des autres volontaires, et défaut d'information immédiate des autorités compétentes, à savoir l'Agence Nationale de Sécurité du Médicament.

Un grand quotidien national a publié quelques semaines plus tard un article révélant que des chiens étaient morts lors des essais animaux, sans que l'on sache la dose qui les avait tués. Il semble encore impossible de prédire ce que l'histoire retiendra de substantiel de cet accident grave. On spéculera 
cependant qu'il se pourrait qu'aucune fraude ni erreur majeure ne fusse identifiée, ni aucune transformation systémique préconisée. Il émergera sans doute une succession de « petits » manquements qui auraient pu rester ignorés sans les aléas de la biologie. Du reste, il est possible que l'on ne connaisse jamais précisément les causes des dégâts humains observés puisqu'en matière de médicament, les échecs cliniques ont ceci de frustrant qu'ils ne permettent pas toujours d'en comprendre les causes [4]. Ils ne délivrent parfois aucune leçon.

Sans doute l'un des rappels majeurs de ce désastre devrait-il concerner l'importance des données précliniques dans le développement des médicaments. Avant les premiers essais de phase I, les molécules ont été testées plus ou moins extensivement chez l'animal. Ce développement préclinique consiste à recourir à différents modèles animaux pour étudier le comportement du médicament candidat. Cette évaluation chez des êtres vivants non humains va s'attacher à décrire la molécule sur le plan de la pharmacologie, de la pharmacocinétique et toxicologique (description des organes cibles et des doses toxiques). D'un point de vue règlementaire, il est exigé que ces essais soient menés sur deux catégories d'animaux : une espèce " rongeur » et une espèce " nonrongeur ».

Cette exigence tire son rationnel de la variabilité de réponse à un médicament d'un système vivant à un autre. Les constantes pharmacologiques et toxicologiques sont dépendantes des modèles animaux sur lesquels les essais vont être menés, ce qui souligne l'importance des essais précliniques pour identifier les futurs effets toxiques cliniques. L'accident récent rappelle bien la criticité des essais animaux.

La sélection de l'espèce « non-rongeur » relève d'un arbitrage majeur. Les ressources bibliographiques, l'historique des pratiques et la sensibilité de l'animal sont des critères décisifs. Le furet est pertinent dans le développement vaccinal car l'animal mime correctement le pouvoir de contagion du virus qu'on retrouvera chez l'homme. Le cochon est très utilisé pour l'étude toxicologique cutanée, sa peau et sa pilo- sité étant proches de celles de l'homme. Et ainsi de suite. Il est ensuite habituel d'étudier la molécule non plus sur un animal mais un modèle animal, c'est-à-dire un animal qui présente la pathologie - spontanée ou induite - la plus similaire possible à la maladie visée chez l'homme. En effet, cette deuxième salve d'évaluation permet souvent une étude pharmacologique plus fine que la précédente, ce qui autorise une meilleure sélection de doses.

Mais les données précliniques ne servent pas seulement à sécuriser la conduite des essais de phase I. Elles cherchent aussi à valider l'efficacité des molécules testées. Il faut rappeler qu'un peu moins de la moitié des essais de phase III sont négatifs, à savoir qu'ils échouent à montrer un rapport bénéfices-risques favorable. Ceci peut être dû à une trop grande toxicité mais c'est souvent lié à une efficacité insuffisante. Et c'est là que le testing animal peut de nouveau être accusé d'avoir été défaillant. Deux types de causes peuvent expliquer cette mauvaise valeur prédictive : un modèle animal inadéquat et une méthodologie insuffisamment rigoureuse qui vient fausser les résultats.

On mesure ainsi qu'autant pour la sécurité que l'efficacité de nos médicaments, les essais précliniques ont une importance vitale. Il ne semblait pas nécessaire d'attendre les conclusions finales des différentes investigations sur l'essai de phase I dont il est question pour s'en souvenir.

Liens d'intérêts : Les auteurs déclarent ne pas avoir de lien d'intérêt.

\section{Références}

1. Butler D, Callaway E (2016) Scientists in the dark after French clinical trial proves fatal. Nature 529:263-4

2. http://blogs.sciencemag.org/pipeline/

3. Carpenter D (2010) Reputation and Power: Organizational Image and Pharmaceutical Regulation at the FDA. Princeton University Press

4. Stockwell B (2011) The Quest for the Cure. Columbia University Press 